\title{
Large and robust electrical spin injection into GaAs at zero magnetic field using an ultrathin $\mathrm{CoFeB/MgO}$ injector
}

\author{
S. H. Liang, ${ }^{1,2}$ T. T. Zhang, ${ }^{3}$ P. Barate, ${ }^{3}$ J. Frougier, ${ }^{4}$ M. Vidal, ${ }^{3}$ P. Renucci, ${ }^{3}$ B. Xu, ${ }^{5}$ H. Jaffrès, ${ }^{4}$ J.-M. George,${ }^{4}$ X. Devaux, ${ }^{1}$ \\ M. Hehn, ${ }^{1}$ X. Marie, ${ }^{3}$ S. Mangin, ${ }^{1}$ H. X. Yang, ${ }^{6}$ A. Hallal, ${ }^{6}$ M. Chshiev, ${ }^{6}$ T. Amand, ${ }^{3}$ H. F. Liu, ${ }^{2}$ D. P. Liu, ${ }^{2}$ X. F. Han, ${ }^{2}$ \\ Z. G. Wang, ${ }^{5}$ and Y. Lu, ${ }^{1, *}$ \\ ${ }^{1}$ Institut Jean Lamour, UMR 7198, CNRS-Nancy Université, BP 239, 54506 Vandouvre, France \\ ${ }^{2}$ Beijing National Laboratory of Condensed Matter Physics, Institute of Physics, Chinese Academy of Sciences, Beijing 100190, China \\ ${ }^{3}$ Université de Toulouse, INSA-CNRS-UPS, LPCNO, 135 avenue de Rangueil, 31077 Toulouse, France \\ ${ }^{4}$ Unité Mixte de Physique CNRS/Thales and Université Paris-Sud 11, 1 avenue A. Fresnel, 91767 Palaiseau, France \\ ${ }^{5}$ Key Laboratory of Semiconductor Materials Science, Institute of Semiconductors, \\ Chinese Academy of Sciences, P.O. Box 912, Beijing 100083, China \\ ${ }^{6}$ SPINTEC, UMR 8191, CEA-INAC/CNRS/UJF-Grenoble 1/G-INP, 38054 Grenoble, France \\ (Received 17 May 2014; revised manuscript received 21 July 2014; published 22 August 2014)
}

\begin{abstract}
Binary information encoded within the spin of carriers can be transferred into corresponding right- or lefthanded circularly polarized photons emitted from an active semiconductor medium via carrier-photon angular momentum conversion. In order to attain maximized spin injection at out-of-plane magnetic remanence, a number of material systems have been explored as possible solid-state spin injectors. However, the circular polarization $\left(P_{\mathrm{C}}\right)$ of emitted light was still limited at $3-4 \%$ at remanence. Here, we demonstrate a sizable electroluminescence circular polarization from a III-V-based spin light-emitting diode at zero magnetic field with a perpendicular spin injector consisting of an ultrathin $\mathrm{CoFeB}$ ferromagnetic layer $(1.2 \mathrm{~nm})$ grown on a $\mathrm{MgO}$ tunnel barrier $(2.5 \mathrm{~nm})$. The maximum value of $P_{\mathrm{C}}$ measured at zero field is as large as $20 \%$ at $25 \mathrm{~K}$ and still $8 \%$ at $300 \mathrm{~K}$. These types of ultrathin perpendicular spin injectors are of great interest (i) to realize the electrical switching of the magnetization of the injector layer owing to the advanced spin-transfer torque properties of the CoFeB layer and (ii) to be directly embedded in optical cavities for spin lasers due to their very low optical absorption loss.
\end{abstract}

DOI: 10.1103/PhysRevB.90.085310

PACS number(s): 72.25.Dc, 78.60.Fi, 75.70.Ak

\section{INTRODUCTION}

Since the discovery of an efficient transfer of a solid-state information stored within ferromagnetic (FM) materials into circularly polarized photons emitted by a spin light-emitting diode (spin-LED) [1-3] via carrier-photon angular momentum conversion, several advanced semiconductor technologies have been proposed. Potential devices, ranging from a memory element with optical readout and optical transport of spin information [4], advanced optical switches [5], circularly polarized single photon emitters for quantum cryptography [6] to chiral analysis [7], and three-dimensional display screens [8], have been anticipated. According to the optical selection rules, $[9,10]$ a conventional spin injector with in-plane magnetization [11-24] cannot satisfy the practical application for quantum well- (QW-) based spin-LEDs because a strong external magnetic field in the range of up to a few Tesla is required to rotate the magnetization into a perpendicular direction. A prerequisite to obtain optimized device functionalities is to promote a robust perpendicular magnetic anisotropy (PMA) medium up to room temperature (RT) to be used as a solid-state FM injector electrode. Good candidates are systems including alternated planes of $3 d / 4 f \mathrm{Fe} / \mathrm{Tb}$ [25,26], $3 d / 5 d \mathrm{Fe} / \mathrm{Pt}$ [27], or $\mathrm{Co} / \mathrm{Pt}[28,29]$, and $3 d / 3 d \mathrm{Co} / \mathrm{Ni}$ multilayers [30]. However, these systems generally suffer from the requirement of a minimum thickness (generally several units of bilayers) grown on a thin oxide layer, which is used as a tunneling barrier to circumvent the conductivity mismatch between metal and

*Corresponding author: yuan.lu@univ-lorraine.fr semiconductor [31]. The large thickness of injector results in a large absorption of light in the near infrared region, e.g., 95\% light is absorbed for $40 \mathrm{~nm} \mathrm{Fe} / \mathrm{Tb}$ multilayers [25,26]. Moreover, in the case of spin-LEDs, there is also a requirement that the first FM atomic plane grown at the interface must possess a robust spin polarization for an efficient spin injection, which is hardly attainable due to the chemical interdiffusion or intermixing in the multilayer systems [29]. Therefore, up to now, the circular polarization $\left(P_{\mathrm{C}}\right)$ of emitted light was still limited at $3-4 \%$ at remanence $[26,27,29,32]$, one of the best results having been obtained by the use of a $7 \mathrm{~nm} \delta-\mathrm{MnGa}$ layer [32].

A series of recent theoretical investigations have proposed that the $\mathrm{Fe}(\mathrm{Co}) / \mathrm{MgO}$ interface itself could provide PMA in the range of magnitude of $1 \mathrm{~mJ} / \mathrm{m}^{2}$, sufficient to reorient the magnetization along the out-of-plane direction [33,34]. This PMA property has been put forward in the case of $\mathrm{CoFeB} / \mathrm{MgO} / \mathrm{CoFeB}$ perpendicular magnetic tunnel junctions (p-MTJs) grown on $\mathrm{SiO}_{2}$ substrate used for spin transfer torque (STT) operations in magnetoresistive random-access memory (MRAM) technologies $[35,36]$. These STT devices display a high tunneling magnetoresistance ratio and good thermal stability, together with low switching current density. In this work, we demonstrate the occurrence of such PMA functionality on semiconducting heterostructures with III-V based spin-LEDs by integrating $\mathrm{CoFeB} / \mathrm{MgO}$ perpendicular spin injectors. Large values of electroluminescence (EL) circular polarization of $20 \%$ at $25 \mathrm{~K}$ and $8 \%$ at $300 \mathrm{~K}$ are measured under zero magnetic field. These results constitute the cornerstone for future implementation of electrical control of circularly polarized light via STT in III-V optical devices 
(a)

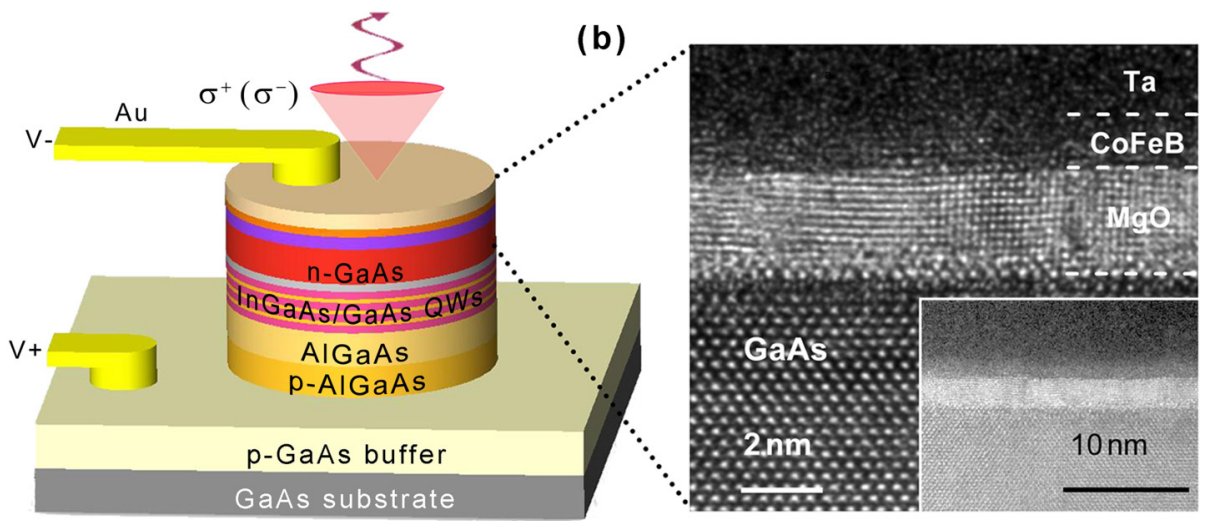

FIG. 1. (Color online) (a) Schematic device structure of spin-LED. (b) HR-TEM image of CoFeB/MgO PMA injector. Inset: low magnification image showing excellent homogeneity and low roughness of structures.

operating at RT, as well as for spin lasers with injectors embedded in the optical cavity that requires very low intracavity optical absorption.

\section{SAMPLE PREPARATION AND STRUCTURAL CHARACTERIZATION}

In our experiments, the $p-i-n$ semiconductor structure of the spin-LED was grown by molecular beam epitaxy (MBE), while the tunnel barrier/ferromagnet contacts were deposited by sputtering. The $p-i-n$ LED device has the following structure sequence: $p$-GaAs:Zn (001) substrate $(p=2 \times$ $\left.10^{19} \mathrm{~cm}^{-3}\right) / 500 \mathrm{~nm} p$-GaAs:Be $\left(p=2 \times 10^{19} \mathrm{~cm}^{-3}\right) / 100$ $\mathrm{nm} \quad p-\mathrm{Al}_{0.3} \mathrm{Ga}_{0.7} \mathrm{As}: \mathrm{Be}\left(p=2 \times 10^{19} \mathrm{~cm}^{-3}\right) / 100 \mathrm{~nm}$ $p-\mathrm{Al}_{0.3} \mathrm{Ga}_{0.7} \mathrm{As}: \mathrm{Be}\left(p=2 \times 10^{18} \mathrm{~cm}^{-3}\right) / 50 \mathrm{~nm}$ undoped $\mathrm{Al}_{0.3} \mathrm{Ga}_{0.7} \mathrm{As} /[15 \mathrm{~nm}$ undoped $\mathrm{GaAs} / 8 \mathrm{~nm}$ undoped $\left.\mathrm{In}_{0.1} \mathrm{Ga}_{0.9} \mathrm{As}\right] \times 3 / 15 \mathrm{~nm}$ undoped $\mathrm{GaAs} / 5 \mathrm{~nm}$ undoped $\mathrm{Al}_{0.3} \mathrm{Ga}_{0.7} \mathrm{As} / 30 \mathrm{~nm}$ undoped GaAs/50 $\mathrm{nm} n$-GaAs:Si $(n=$ $\left.1 \times 10^{16} \mathrm{~cm}^{-3}\right)$. The LED surface was passivated with arsenic in the III-V MBE chamber. The intended design of the $3 \mathrm{QWs}$ structure for LED is to obtain stronger EL intensity, especially at RT. Another important reason is that the surface root mean square (RMS) roughness of $3 \mathrm{QWs}-\mathrm{LED}(0.21 \mathrm{~nm}$ measured by atomic force microscopy) is found to be much better than that of the single QW-LED $(0.33 \mathrm{~nm})$, which is also a critical factor to obtain a continuous ultrathin $\mathrm{CoFeB}$ layer. The sample was then transferred through the air into a magnetron sputteringMBE interconnected system to grow the $\mathrm{CoFeB} / \mathrm{MgO}$ spin injector. The arsenic capping layer was first desorbed at $300^{\circ} \mathrm{C}$ by monitoring in situ reflection high energy electron diffraction patterns in the MBE chamber, and then the sample was transferred to the sputtering chamber to grow the spin injector. The spin injector, grown at RT, consists of a $2.5 \mathrm{~nm} \mathrm{MgO}$ tunnel barrier and a thin $\mathrm{Co}_{40} \mathrm{Fe}_{40} \mathrm{~B}_{20} \mathrm{FM}$ layer (1.1-1.7 nm). Finally, $5 \mathrm{~nm}$ Ta was deposited to prevent oxidation. The $300 \mu \mathrm{m}$ diameter circular mesas were then processed using standard UV photolithography and etching techniques. In the end, the processed wafers were cut into small pieces to perform rapid temperature annealing (RTA) at different temperatures for $3 \mathrm{~min}$. The RTA procedure is a good way to promote PMA of $\mathrm{CoFeB}$ [37], while almost keeping no change to the LED optical characteristics. High-resolution transmission electron microscopy (HR-TEM) and high-resolution scanning transmission electron microscopy (HR-STEM) studies were performed by using a JEOL ARM200 cold field-emission gun working at $200 \mathrm{kV}$.

The whole structure of the sample is schematically shown in Fig. 1(a). The interface of the spin injector consisting of a $1.2 \mathrm{~nm} \mathrm{CoFeB}$ layer was annealed at $300{ }^{\circ} \mathrm{C}$ and then investigated by HR-TEM and HR-STEM. The low magnification HR-TEM image [inset of Fig. 1(b)] reveals a good homogeneity and a very low roughness of $\mathrm{MgO}$ on GaAs. Moreover, the continuity of the $1.2 \mathrm{~nm} \mathrm{CoFeB} \mathrm{layer} \mathrm{can}$ also be validated in this picture. From the high magnification HR-TEM image [Fig. 1(b)], a $\mathrm{MgO}$ (001) texture is clearly revealed with an abrupt interface to both the $\mathrm{CoFeB}$ and GaAs layers, whereas the interface of $\mathrm{Ta} / \mathrm{CoFeB}$, whose quality is less important for PMA, appears rather diffusive. For HR-STEM investigations, high-angle annular dark-field detector (acceptance angle 68-280 mrad), low-angle annular dark-field detector (LAADF; acceptance angle 30-120 mrad) and bright-field detector (BF; acceptance angle 0-5.6 mrad) were combined for simultaneous image observation with a $\mathrm{Z}$ contrast. As shown in the HR-STEM images [Figs. 2(a) and 2(b)], the phase distribution at the interface can be directly deduced from the image contrast. From the BF image [Fig. 2(b)], we can also identify a continuous ultrathin layer of $\mathrm{CoFeB}$ but with a rough $\mathrm{Ta} / \mathrm{CoFeB}$ interface, indicating an intermixing or diffusion of $\mathrm{Ta}$ in the $\mathrm{CoFeB}$ layer after annealing. The mean thickness of the $\mathrm{CoFeB}$ layer was

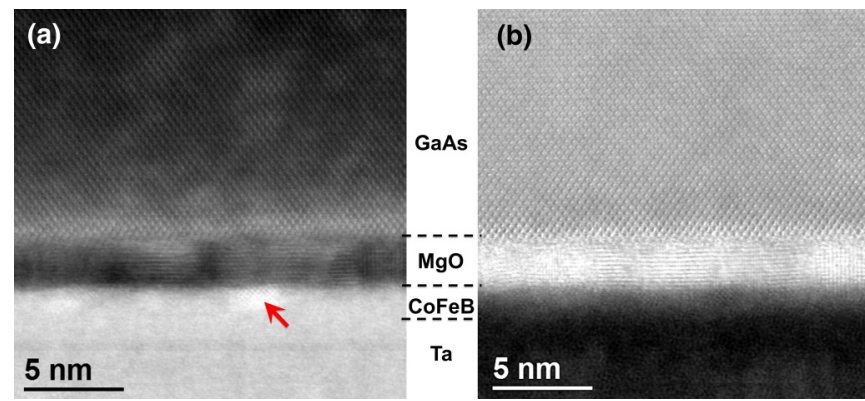

FIG. 2. (Color online) HR-STEM images from the same observed zone. (a) LAADF image and (b) BF image. The red arrow on the LAADF image points out the beginning of the crystallization of the $\mathrm{CoFeB}$ phase starting from the $\mathrm{CoFeB} / \mathrm{MgO}$ interface. 

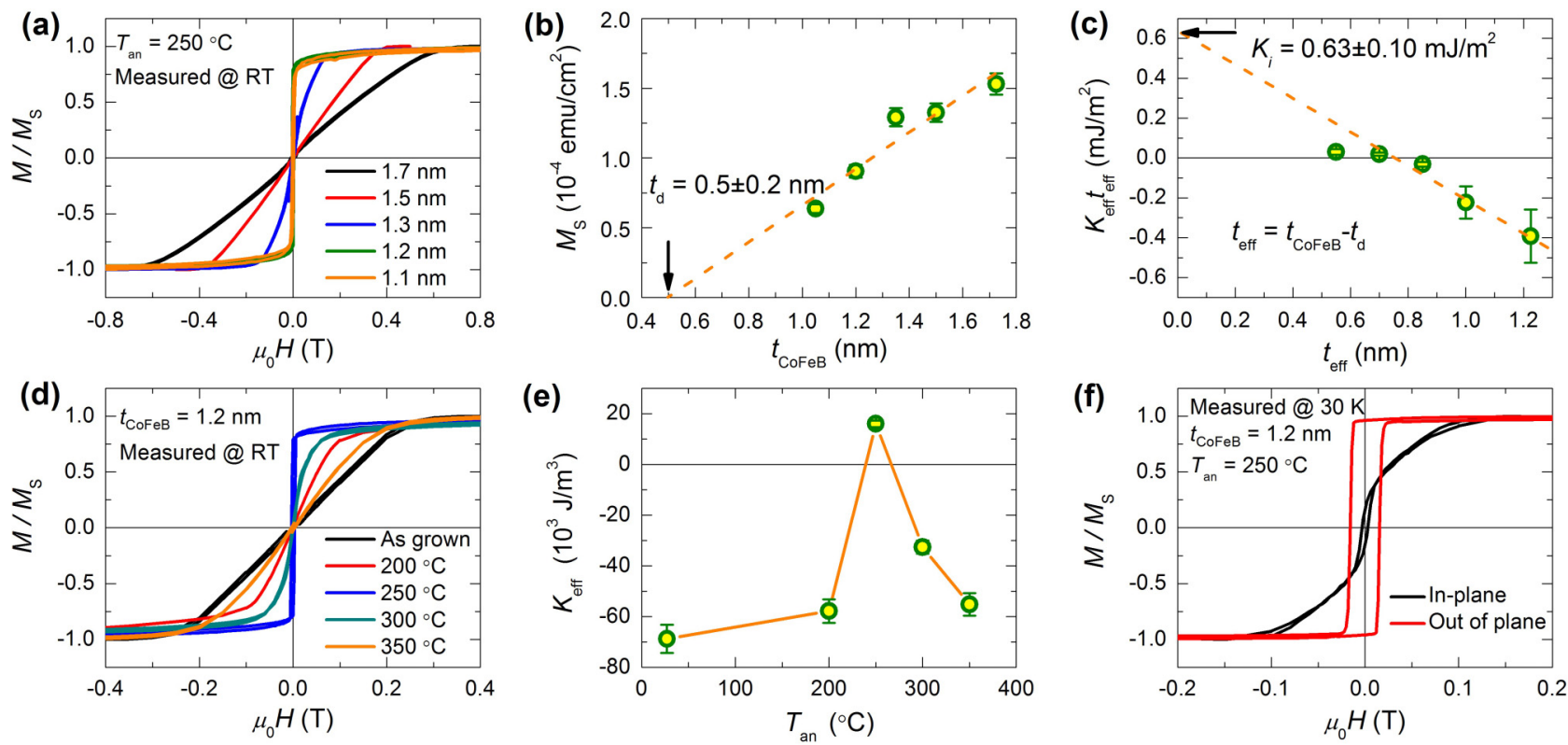

FIG. 3. (Color online) (a) RT out-of-plane $M-H$ curves for spin injectors with different $\mathrm{CoFeB}$ thicknesses with $T_{\text {an }}=250^{\circ} \mathrm{C}$. (b) Extrapolation of CoFeB magnetic dead layer $t_{\mathrm{d}}$ from the CoFeB thickness-dependent saturation magnetization $M_{\mathrm{s}}$. (c) $t_{\mathrm{eff}}$ dependence of the product of $K_{\text {eff }}$ and $t_{\text {eff }}$, where the intercept to the vertical axis of the linear extrapolation corresponds to $K_{\mathrm{i}}$. (d) RT out-of-plane $M-H$ curves for spin injectors with $1.2 \mathrm{~nm} \mathrm{CoFeB}$ before and after different temperature annealing. (e) $K_{\text {eff }}$ as a function of annealing temperature. (f) $M-H$ curves at $30 \mathrm{~K}$ for spin injector with $1.2 \mathrm{~nm} \mathrm{CoFeB}$ and $T_{\mathrm{an}}=250^{\circ} \mathrm{C}$ for in-plane and out-of-plane configurations.

estimated to be $1.2 \pm 0.3 \mathrm{~nm}$. Moreover, the red arrow on the LAADF image [Fig. 2(a)] points out the beginning of the crystallization of the $\mathrm{CoFeB}$ phase, starting from the $\mathrm{CoFeB} / \mathrm{MgO}$ interface.

\section{MAGNETIC PROPERTIES OF SPIN INJECTOR}

To obtain an ultrathin CoFeB layer with PMA on GaAs, we have optimized $\mathrm{CoFeB}$ thickness and annealing temperature $\left(T_{\text {an }}\right)$ by superconducting quantum interference device (SQUID) magnetometry measurements. Figure 3(a) displays the out-of-plane magnetization vs external magnetic field $(M-H)$ curves for annealed spin injectors $\left(T_{\text {an }}=250^{\circ} \mathrm{C}\right)$ with different $\mathrm{CoFeB}$ thicknesses. The saturation field is found to rapidly decrease on reducing $\mathrm{CoFeB}$ thickness. When the thickness is lower than $1.2 \mathrm{~nm}, \mathrm{CoFeB}$ possesses a remanent out-of-plane magnetization signifying the occurrence of PMA. This behavior can be easily understood from the competition between the bulk in-plane shape anisotropy $\left(K_{\mathrm{b}}\right)$ and the interface anisotropy $\left(K_{\mathrm{i}}\right)$ scaling with a resulting " $1 / t$ " volume anisotropy. From the analysis of saturation magnetization $\left(M_{\mathrm{s}}\right)$ vs $\mathrm{CoFeB}$ thickness and the loss of $M_{\mathrm{s}}$ at smaller thickness [Fig. 3(b)], a thick magnetic dead layer $(0.5 \pm 0.2 \mathrm{~nm})$ of $\mathrm{CoFeB}\left(t_{\mathrm{d}}\right)$ is revealed in good agreement with other reported results [38]. This dead layer could be attributed to the intermixing at the top $\mathrm{Ta} / \mathrm{CoFeB}$ interface during deposition or upon annealing [39], which is also evidenced from the diffusive interface on the TEM images. The PMA energy density per unit volume $\left(K_{\text {eff }}\right)$ varies with the effective CoFeB thickness $\left(t_{\mathrm{eff}}=t_{\mathrm{CoFeB}}-t_{\mathrm{d}}\right)$ [Fig. 3(c)] and can be obtained from the integrated difference between the out-of-plane and in-plane $M-H$ curves. When $K_{\text {eff }}>0$, the CoFeB is characterized by a perpendicular easy axis of magnetization, because $K_{\mathrm{i}}$ can be obtained from the intercept of $K_{\mathrm{eff}} \cdot t_{\mathrm{eff}} \mathrm{vs} t_{\mathrm{eff}}$ linear fitting. The value found from our results is about $0.63 \pm 0.10 \mathrm{~mJ} / \mathrm{m}^{2}$, which is lower than the value of $1.3 \mathrm{~mJ} / \mathrm{m}^{2}$ given by Ikeda et al. [35] for metallic MTJ.

A precise control over the annealing temperature is also an important factor to obtain a good PMA property. Figure 3(d) compares the $M-H$ curves for different $T_{\text {an }}$ in $1.2 \mathrm{~nm} \mathrm{CoFeB} / \mathrm{MgO}$, with corresponding $K_{\text {eff }}$ vs $T_{\text {an }}$ plotted in Fig. 3(e). The optimized annealing temperature is found to be around $250^{\circ} \mathrm{C}$. Below or above this temperature, PMA is much reduced, and the magnetization rotates back along the in-plane direction. As already investigated theoretically by Yang et al. [33], the PMA is very sensitive to the $\mathrm{Fe}(\mathrm{Co}) / \mathrm{MgO}$ interface chemical structure. The improvement of PMA at $T_{\text {an }}$ up to $250^{\circ} \mathrm{C}$ could be attributed to an optimization of the chemical structure at $\mathrm{CoFeB} / \mathrm{MgO}$ interface [40]. (Please see Sec. IV for more detailed analysis of the influence of interface structure on PMA.) When $T_{\text {an }}$ exceeds $250^{\circ} \mathrm{C}$, Ta species start to diffuse through the ultrathin $\mathrm{CoFeB}$ to $\mathrm{MgO}$ interface and significantly damage PMA [41]. As our best EL results are measured at low temperature, Fig. 3(f) shows the in-plane and out-of-plane $M-H$ curves at $30 \mathrm{~K}$ for the perpendicular injector with optimized conditions for $t_{\mathrm{CoFeB}}=1.2 \mathrm{~nm}$ and $T_{\mathrm{an}}$ $=250^{\circ} \mathrm{C}$. We can observe a clear perpendicular easy axis with out-of-plane coercivity $\mu_{0} H_{\mathrm{C}}=20 \mathrm{mT}$ and in-plane saturation field $\mu_{0} H_{\mathrm{K}}=150 \mathrm{mT}$. The perpendicular anisotropy energy density $K_{\text {eff }}$ is then determined to be $4.6 \times 10^{4} \mathrm{~J} / \mathrm{m}^{3}$.

\section{PERPENDICULAR MAGNETIC ANISOTROPY}

In the light of recent theoretical investigations [42], and in order to have a detailed understanding of the origin of PMA, we have performed $a b$ initio calculations using the Vienna 
(a) $\quad \Delta \mu\left(10^{-2} \mu_{\mathrm{B}}\right)$

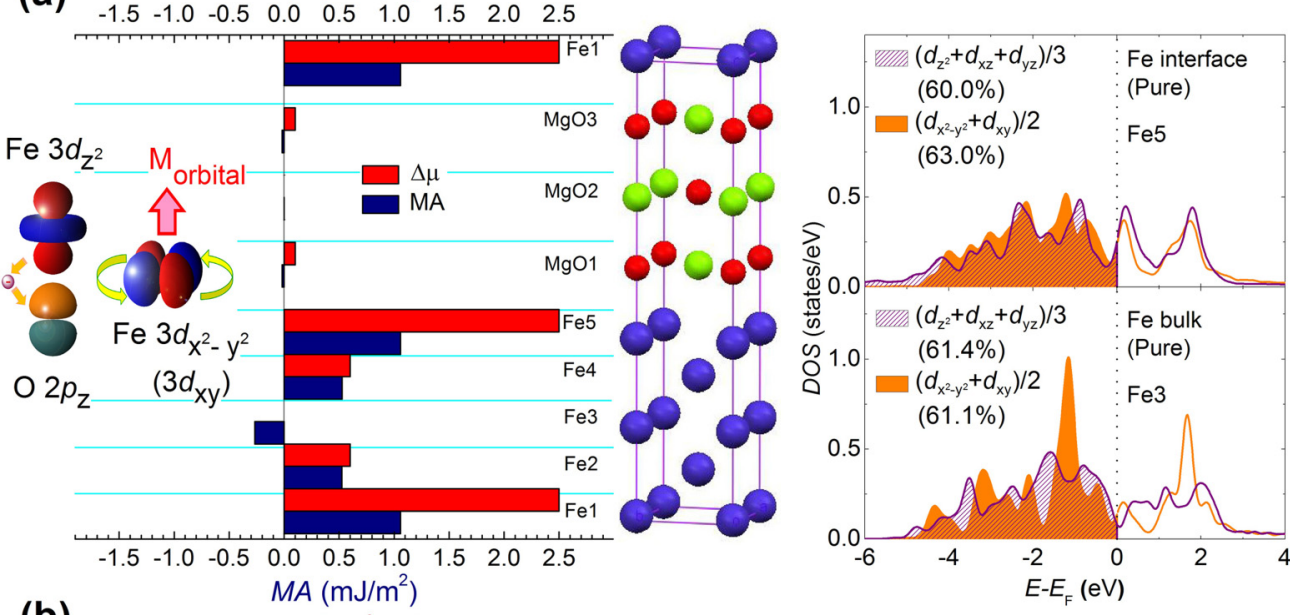

(b)

$$
\Delta \mu\left(10^{-2} \mu_{\mathrm{B}}\right)
$$
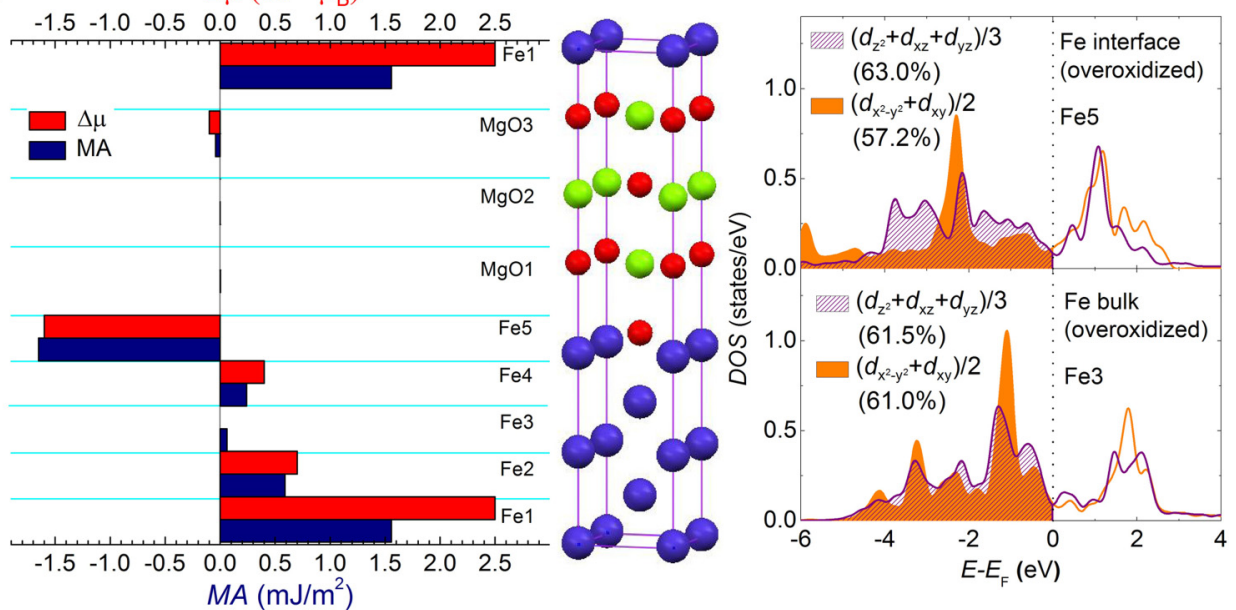

(c) $\Delta \mu\left(10^{-2} \mu_{\mathrm{B}}\right)$
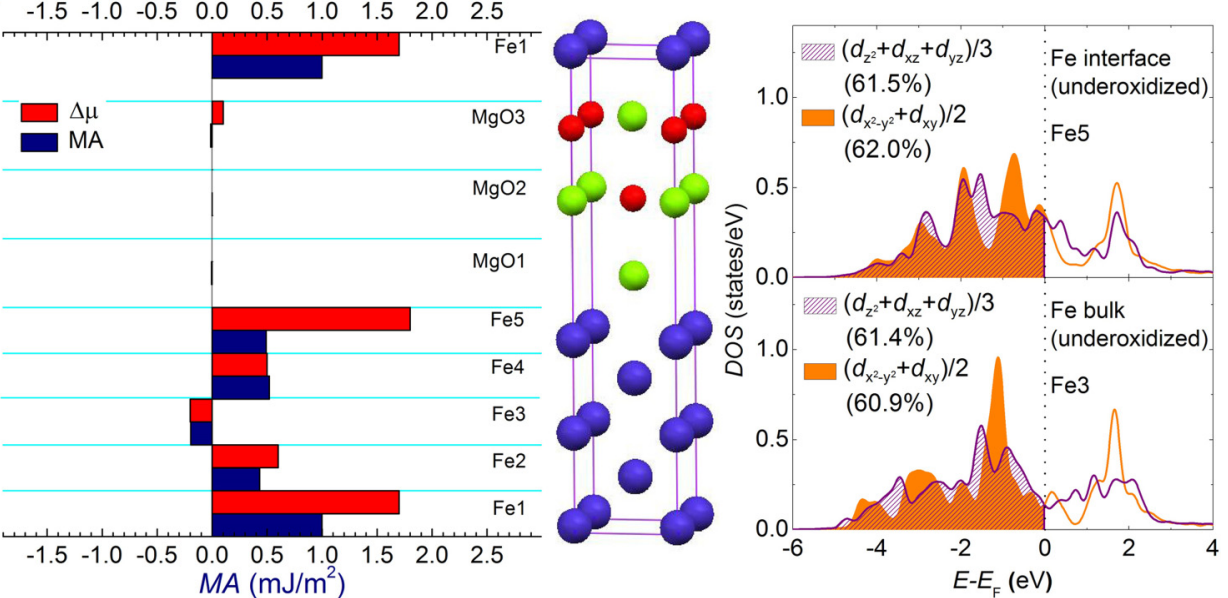

FIG. 4. (Color online) (a) Left: layer-resolved orbital moment anisotropy $(\Delta \mu)$ along with MA energy. Middle: schematics of the calculated crystalline structures. Fe, $\mathrm{Mg}$, and $\mathrm{O}$ are represented by blue, green, and red balls, respectively. Right: DOS with SOC for averaged Fe $3 d$ out-of-plane $\left(3 d_{\mathrm{z}^{2}}+3 d_{\mathrm{xz}}+3 d_{\mathrm{yz}}\right)$ and in-plane orbitals $\left(3 d_{\mathrm{x}^{2}-\mathrm{y}^{2}}+3 d_{\mathrm{xy}}\right)$ with Fe both at the interface (Fe5) and in the bulk (Fe3). Inset: a simple picture showing that the origin of PMA comes from the hybridization of Fe out-of-plane orbitals $\left(3 d_{\mathrm{z}^{2}}, 3 d_{\mathrm{xz}}\right.$ and $\left.3 d_{\mathrm{yz}}\right)$ and $\mathrm{O} 2 p_{\mathrm{z}}$ orbitals, which leads to an uncompensated charge occupation in Fe in-plane orbitals $\left(3 d_{\mathrm{x}^{2}-\mathrm{y}^{2}}, 3 d_{\mathrm{xy}}\right)$ and results in an enhanced out-of-plane orbital moment for PMA. Two other different interface structures: (b) overoxidized and (c) underoxidized geometries.

$A b$ initio Simulation Package [43-45] with the generalized gradient approximation [46] and projector-augmented wave potentials $[47,48]$. The system calculated is composed of five Fe layers sandwiched between three $\mathrm{MgO}$ layers at both sides, as shown in Fig. 4(a) (middle). A $19 \times 19 \times 3$ k-point mesh was used in our calculations. Calculations were performed in three steps. First, full structural relaxation in shape and volume was performed until the forces become lower than $0.001 \mathrm{eV} / \AA$ 
for determining the most stable interfacial geometries. Next, the Kohn-Sham equations were solved with no spin-orbit coupling (SOC) taken into account to determine the charge distribution of the system ground state. Finally, the density of states (DOS), orbital moment anisotropy $(\Delta \mu)$, and magnetic anisotropy (MA) energy were calculated from corresponding orbital moment and energy of the system for out-of-plane and in-plane magnetization orientation with SOC included.

We then explain the origin of PMA in our system in the following pedagogical way. In bulk Fe with bcc structure, the charge distribution in the $3 d$ shell is almost isotropic, as well as the resulting average orbital moment. The orbital moment acquired in the plane of the layer exactly compensates that acquired along the out-of-plane direction by equal filling of the corresponding orbitals. This produces zero orbital moment anisotropy $(\Delta \mu)$, as shown on the left in Fig. 4(a) for the bulk atom $\mathrm{Fe} 3$. In contrast to bulk $\mathrm{Fe}$ film, the $\mathrm{Fe} / \mathrm{MgO}$ interface exhibits a strong uniaxial character. The out-of-plane Fe $3 d_{\mathrm{z}^{2}}$ orbital strongly bonds to the $\mathrm{O} 2 p_{\mathrm{z}}$ orbital, and it introduces a significant charge transfer from $\mathrm{Fe}$ to $\mathrm{O}$ orbitals [inset of Fig. 4(a) on the left]. This results in a lack of electrons within the $\mathrm{Fe} 3 d_{\mathrm{z}^{2}}, 3 d_{\mathrm{xz}}$, and $3 d_{\mathrm{yz}}$ out-of-plane orbitals compared to the Fe in-plane orbitals $\left(3 d_{\mathrm{x}^{2}-\mathrm{y}^{2}}\right.$ and $\left.3 d_{x y}\right)$. As a consequence, an enhanced out-of-plane orbital moment occurs from the uncompensated in-plane orbitals and generates a sizable PMA once the SOC is introduced. To better understand this simple explanation of PMA origin, we show [on the right in Fig. 4(a)] the DOS with SOC of averaged Fe $3 d$ out-of-plane $\left(d_{\mathrm{z}^{2}}+d_{\mathrm{xz}}+d_{\mathrm{yz}}\right)$ and in-plane orbitals $\left(d_{\mathrm{x}^{2}-\mathrm{y}^{2}}+d_{\mathrm{xy}}\right)$ for Fe both at the interface and in the bulk (spin up and down are mixed due to SOC). From the integration of the occupied states below Fermi level $\left(E_{\mathrm{F}}\right)$, we found a difference of about $3 \%$ between respective out-of-plane and in-plane orbitals for $\mathrm{Fe}$ atom bound to $\mathrm{O}$ at the interface (Fe5), while almost no difference appears for Fe in the bulk $(\mathrm{Fe} 3)$. This gives an unbalanced orbital moment anisotropy $\left(\Delta \mu \approx 0.03 \mu_{\mathrm{B}}\right)$ associated with the magnetization or spins in the out-of-plane direction for the interfacial atom Fe5 (and Fe1). According to Bruno's model [49], the anisotropy energy $\left(\Delta E_{\mathrm{SO}}\right)$ can be obtained by $\Delta E_{\mathrm{SO}}=\xi_{\mathrm{SO}} \frac{\Delta \mu}{4 \mu_{\mathrm{B}}}$ considering that the spin moment remains unaffected at the interface, where $\xi_{\text {SO }}$ is the SOC parameter. The interface anisotropy $K_{\mathrm{i}}$ can then be qualitatively estimated from $\Delta E_{\mathrm{SO}}$ in the range of $1 \mathrm{~mJ} / \mathrm{m}^{2}$, which is in good agreement with the quantitatively calculated MA energy [on the left in Fig. 4(a)].

As we discussed above, the PMA originates from a net uncompensated out-of-plane orbital moment at the $\mathrm{Fe}(\mathrm{Co}) / \mathrm{MgO}$ interface. Such sizable anisotropy of orbital moment is expected to be very sensitive to the interface atomic configuration and bonding. To further prove our explanation, we have calculated the DOS, $\Delta \mu$ and MA for overoxidized (with $\mathrm{O}$ inserted at the interfacial magnetic layer) and underoxidized (Mg-terminated) interfaces, as shown in Figs. 4(b) and 4(c), respectively. The details of calculation are available in our previously published work [33]. The calculated averaged DOS represents Fe $3 d$ out-of-plane $\left(3 d_{\mathrm{z}^{2}}+3 d_{\mathrm{xz}}+3 d_{\mathrm{yz}}\right)$ and in-plane orbitals $\left(3 d_{\mathrm{x}^{2}-\mathrm{y}^{2}}+3 d_{\mathrm{xy}}\right)$ according to our convention for interfacial $(\mathrm{Fe} 5)$ and bulk iron $(\mathrm{Fe} 3)$ atoms. One can see that for all cases, the almost equal in-plane and out-of-plane orbital occupation in bulk Fe results in rather small orbital moment anisotropy and MA energy. However, the situation is quite different at the interface. We have found a difference of about $3 \%$ with higher in-plane orbital occupation for pure Fe interface, while a $5 \%$ difference with higher out-of-plane orbital occupation for the overoxidized interface, and only a $0.5 \%$ difference with higher in-plane orbital occupation for the underoxidized interface. As the PMA originates from the orbital moment anisotropy [49], which is related to the orbital occupation, this results in a strong PMA at the pure $\mathrm{Fe}$ interface, a complete loss of PMA for overoxidized interface and a much reduced PMA at the underoxidized interface. This model is in qualitative agreement with the calculated MA values (total energy difference between in-plane and out-of-plane magnetizations), as shown in Fig. 4 (left panel).

Our simple model can explain qualitatively the obtained results. Concerning the overoxidized $\mathrm{Fe} / \mathrm{MgO}$ interface, since the bonding mechanism with $\mathrm{O}$ species occurs now along both out-of-plane and in-plane directions, the stronger bonding along in-plane direction results in a higher out-of-plane orbital occupation. This completely changes the interface anisotropy and promotes an in-plane magnetization orientation at the interface. On the other hand, for the underoxidized interface, a lack of bonding with $\mathrm{O}$ species makes an almost equivalent orbital occupation for in-plane and out-of-plane orbitals, which also reduces the PMA. Therefore, our simple model from analysis of the anisotropy of orbital charge occupation and the orbital moment anisotropy can well explain the origin of PMA and give a very simple and direct image to understand the PMA nature.

\section{ELECTROLUMINESCENCE MEASUREMENTS}

\section{A. Magnetic field dependence}

In the following, we focus on EL measurements on an optimized sample. For the polarization-resolved EL measurements, the spin-LED was placed into a Helmholtz split magnetic coil providing a maximum magnetic field of $0.8 \mathrm{~T}$ normal to the sample plane. The EL signal was detected in the Faraday geometry. The spectral resolution of the setup is $2 \mathrm{meV}(1.3 \mathrm{~nm})$. The EL circular polarization $P_{\mathrm{C}}$ was analyzed through a $\lambda / 4$ wave plate and a linear analyzer. $P_{\mathrm{C}}$ is defined as $P_{\mathrm{C}}=\left(I^{\sigma+}-I^{\sigma-}\right) /\left(I^{\sigma+}+I^{\sigma-}\right)$, where $I^{\sigma+}$ and $I^{\sigma-}$ are the intensities of the right and left circularly polarized components of the luminescence, respectively. A typical EL spectrum acquired at $25 \mathrm{~K}$ under a bias $\left(V_{\text {bias }}\right)$ of $2.30 \mathrm{~V}$ with a current density $(J)$ of $2.8 \mathrm{~A} / \mathrm{cm}^{2}$ is shown in the top of Fig. 5(a) for $\mu_{0} H=0 \mathrm{~T}$. In this spectrum, we can observe a main peak located at about $873 \mathrm{~nm}$ corresponding to the heavy exciton line, with a small shoulder at about $870 \mathrm{~nm}$. The multipeak feature could be attributed to a slightly different indium concentration for the three InGaAs QWs, as well as the possible bound exciton at low temperature [50-52]. The striking feature is that we can have a large difference of the EL intensities for right $\left(I^{\sigma+}\right)$ and left $\left(I^{\sigma-}\right)$ circularly polarized components at zero field. The EL circular polarization $\left(P_{\mathrm{C}}\right)$ can be determined from the main peak difference for $I^{\sigma+}$ and $I^{\sigma-}$ to be about $13 \%$. To further confirm that this feature originates from the perpendicular spin injector, we have measured the $P_{\mathrm{C}}$ 
(a)
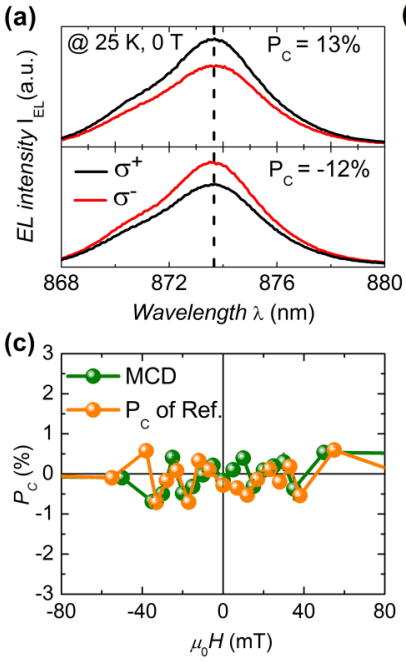

(b)

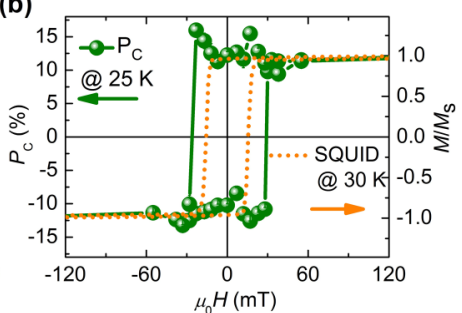

(d)

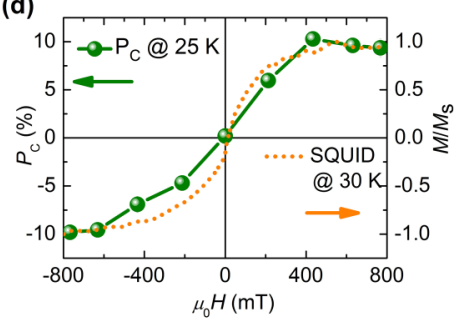

FIG. 5. (Color online) (a) EL spectra at $25 \mathrm{~K}$ with zero magnetic field for the optimized PMA condition sample for $\sigma^{+}$and $\sigma^{-}$ polarizations when field is swept from positive to negative direction (top) and from negative to positive direction (bottom) in the hysteresis loop. (b) and (d) $P_{\mathrm{C}}$ as a function of out-of-plane magnetic field measured at $25 \mathrm{~K}\left(V_{\text {bias }}=2.30 \mathrm{~V}, J=2.8 \mathrm{~A} / \mathrm{cm}^{2}\right)$ for spin-LED with $1.2 \mathrm{~nm} \mathrm{CoFeB/MgO}$ injectors, which are compared to the corresponding out-of-plane $M-H$ hysteresis loop at $30 \mathrm{~K}$ by SQUID. (b) Optimized PMA injector. (d) As-grown injector. (c) MCD induced by the ultrathin $\mathrm{CoFeB}$ electrode as a function of magnetic field at $25 \mathrm{~K}$, determined by measuring the circular polarization (green circles) of the luminescence emitted under linearly polarized excitation of the spin-LED by a He-Ne laser. $P_{\mathrm{C}}$ of EL (orange circles) for a reference sample without $\mathrm{CoFeB}$ layer (replaced by a nonmagnetic Ta layer), as a function of magnetic field at $25 \mathrm{~K}$.

variation at different magnetic fields. As shown in Fig. 5(b), $P_{\mathrm{C}}$ exhibits a clear hysteresis loop feature, with an almost constant value around $13 \%$ at saturation and changes its sign rapidly at $\mu_{0} H= \pm 30 \mathrm{mT}$. The bottom of Fig. 5(a) displays the spectrum at $\mu_{0} H=0 \mathrm{~T}$ when the field is swept from negative to positive direction. The hysteresis loop of $P_{\mathrm{C}}$ fairly matches the SQUID hysteresis loop acquired at $30 \mathrm{~K}$ on an unpatterned sample [Fig. 5(b)]. The slight difference in the coercivity could be due to a slightly different calibration of temperature in the two systems or a small different effective RTA annealing temperature for the two measured samples.

To exclude any artificial effect for the measured circular polarization at remanence (zero magnetic field), we have performed two complementary measurements. One is the evaluation of magnetic circular dichroism (MCD) effect in order to check the differential absorption of respective left and right circularly polarized light [26] through the CoFeB FM layer in our sample. With linearly polarized excitation light, we have recorded the MCD signal by photoluminescence (PL) with different magnetic fields. As shown in Fig. 5(c), the MCD effect from the PMA spin-LED sample is lower than $1 \%$ in all investigated field ranges, and this means that the large $P_{\mathrm{C}}$ is really due to the circularly polarized light emitted from the radiative recombination of the spin-polarized electron with unpolarized holes in the QW. The other measurement is to exclude artificial effects, such as Zeeman splitting in the QW

[13] by EL characterization of a reference sample without the $\mathrm{CoFeB}$ layer, which can allow us to verify the origin of this spin-polarized injection of electrons. The reference sample has almost the same structure except that the ultrathin $\mathrm{CoFeB}$ layer is now replaced by a nonmagnetic Ta layer in contact with $\mathrm{MgO}$. As shown in Fig. 5(c), $P_{\mathrm{C}}$ from the reference sample also shows less than $1 \%$ in all investigated field range. This makes a strong argument that the large $P_{\mathrm{C}}$ we have observed is really due to the spin-polarized electron injected from the ultrathin CoFeB layer with PMA.

What then is the signature of the polarization-resolved EL for an in-plane magnetization injector, such as the as-grown CoFeB/MgO injector? In Fig. 5(d), we show $P_{\mathrm{C}}$ vs field for the same spin-LED structure before annealing. As expected, $P_{\mathrm{C}}$ increases linearly with the field before reaching its saturation value $(10 \%)$ at about $0.4 \mathrm{~T}$. The variation of $P_{\mathrm{C}}$ vs field matches well with the corresponding variation of the out-of-plane magnetization. Indeed, in this configuration, $P_{\mathrm{C}}$ tracks the continuous rotation of magnetization direction from in-plane to out-of-plane, as expected from the optical selection rules in the QW $[9,10]$.

\section{B. Temperature dependence}

Another very interesting behavior is that the PMA property of our spin injector can even persist up to RT. The inset of Fig. 6(a) shows the EL spectra with different circular polarizations at $300 \mathrm{~K}$ under zero field. A clear difference of $I^{\sigma+}$ and $I^{\sigma-}$ components allows us to obtain $P_{\mathrm{C}}=8 \%$ at RT. The $P_{\mathrm{C}}$ hysteresis loop is also in good agreement with the RT $M-H$ hysteresis loop [Fig. 6(a)]. Although the out-of-plane coercivity $\mu_{0} H_{\mathrm{C}}$ is reduced at about $5 \mathrm{mT}$, it is sufficient to obtain an almost full remanent magnetization. To further investigate the temperature dependence of the spin injection efficiency with PMA injectors, we have plotted in Fig. 6(b) the temperature dependence of $P_{\mathrm{C}}$ without field and with $0.4 \mathrm{~T}$ field, which is sufficiently large to ensure the saturation of the out-of-plane magnetization. We have observed a nonmonotonic variation of $P_{\mathrm{C}}$ as a function of $T$ : first a decrease of $P_{\mathrm{C}}$, followed by a low varying regime above $100 \mathrm{~K}$. A notable feature is the very similar evolution for $P_{\mathrm{C}}$ at both $\mu_{0} H=0 \mathrm{~T}$ and $\mu_{0} H=0.4 \mathrm{~T}$. This behavior confirms that the PMA is strong enough to persist up to RT.

The nonmonotonic evolution of $P_{\mathrm{C}}$ likely reflects physical effects inherent to the semiconductor heterostructures rather than to the property of $\mathrm{CoFeB} / \mathrm{MgO}$ injector itself. In order to check this assumption, we have performed time-resolved PL (TRPL) measurements [53] on an identical $p-i-n$ LED. Please see the Appendix for detailed information on TRPL measurements and the method to extract the spin-relaxation time $\left(\tau_{\mathrm{s}}\right)$ and carrier lifetime $(\tau)$. As known, the optical circular polarization $P_{\mathrm{C}}$ directly equals the injected electron spin polarization $P_{\mathrm{E}}$ except for a renormalization factor (called $F$ ), which takes into account possible electron spin relaxation mechanisms during its lifetime in the QW [20,54]. The spin relaxation time $\tau_{\mathrm{s}}$, as well as the carrier lifetime $\tau$, extracted from the TRPL measurements, are presented in Fig. 6(c). A relatively weak variation of the carrier lifetime $\tau$ (of the order of $100 \mathrm{ps)} \mathrm{vs} T$ can be highlighted, whereas a strong thermal variation of the spin lifetime $\tau_{\mathrm{s}}$ is evidenced with an initially 

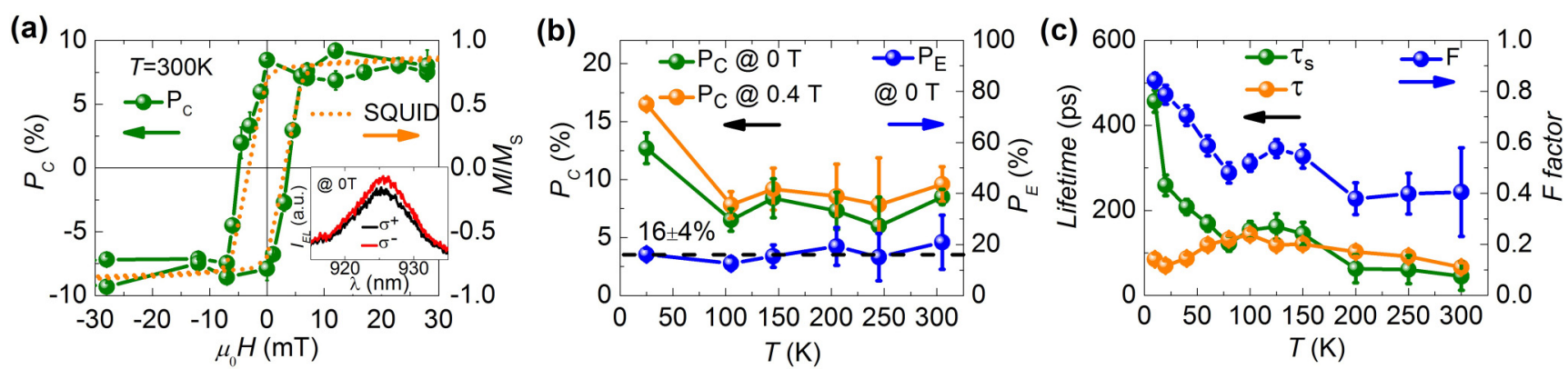

FIG. 6. (Color online) (a) $P_{\mathrm{C}}$ as a function of out-of-plane magnetic field measured at $300 \mathrm{~K}$ ( $\left.V_{\text {bias }}=2.30 \mathrm{~V}\right)$ for the spin-LED with optimized PMA injector, which is compared to the out-of-plane $M-H$ hysteresis loop at $300 \mathrm{~K}$ by SQUID. Inset: EL spectra at $300 \mathrm{~K}$ with zero magnetic field for $\sigma^{+}$and $\sigma^{-}$polarizations. (b) Temperature dependence of $P_{\mathrm{C}}$ without magnetic field and with $0.4 \mathrm{~T}$ field. Temperature dependence of $P_{\mathrm{E}}$ is calculated by $P_{\mathrm{E}}=P_{\mathrm{C}} / F$ from the data without field. (c) Temperature dependence of spin lifetime ( $\tau_{\mathrm{s}}$ ), carrier lifetime $(\tau)$, and $F$ factor deduced from the TRPL measurements.

fast falloff, followed by a smoother decline. The large increase of spin lifetime at low temperature could be attributed to the spatial localization of excitons due to the inhomogeneity of QWs. When the temperature increases, the spin relaxation is then dominated by Dyakonov-Perel (DP) mechanism [10], which gives a small variation of $\tau_{\mathrm{s}}$ up to RT. We also plotted in Fig. 6(c) the temperature dependence of the renormalization factor $F=1 /\left(1+\tau / \tau_{\mathrm{s}}\right)$ that links $P_{\mathrm{C}}$ to the true electrically injected electron spin polarization $P_{\mathrm{E}}$, according to $P_{\mathrm{C}}(T)=$ $F(T)^{*} P_{\mathrm{E}}[20,54]$. The $F$ factor vs $T$ behavior mimics the variation of $P_{\mathrm{C}}$ vs $T$, which results in an overall small variation of $P_{\mathrm{E}}$ vs $T . P_{\mathrm{E}}$ is found to be almost temperature insensitive at about $16 \pm 4 \%$ [Fig. 6(b)]. This also confirms a thermal stability of our PMA spin injector with high Curie temperature FM layer. Note this value of $P_{\mathrm{E}}$ is an average value on the three QWs and does not reflect directly the exact spin polarization at $\mathrm{MgO} / \mathrm{GaAs}$ interface. The evaluation of spin losses between this interface and the QWs will require further investigation, for example, by probing different samples where the QWs would be located at different distances from the interface, as in Ref. [55].

\section{Bias dependence}

Finally, we have measured $P_{\mathrm{C}}$ as a function of the applied bias at $25 \mathrm{~K}$ under zero field. As shown in Fig. 7, $P_{\mathrm{C}}$ is

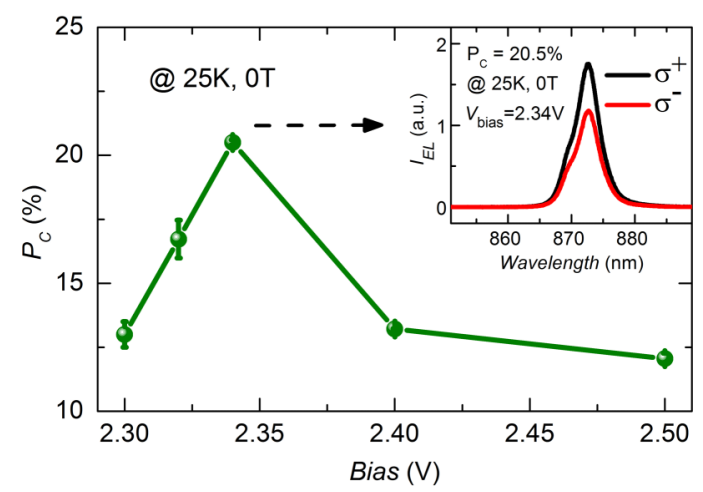

FIG. 7. (Color online) $P_{\mathrm{C}}$ as a function of applied bias for the optimized PMA condition sample. Inset: EL spectra at $25 \mathrm{~K}$ with zero magnetic field for $\sigma^{+}$and $\sigma^{-}$polarizations under a bias of $2.34 \mathrm{~V}$. found to be strongly dependent on the bias. Our maximum $P_{\mathrm{C}}$ in remanence can even reach $20 \%$ at the optimal bias of $2.34 \mathrm{~V}$. The corresponding polarization-resolved EL spectra are shown in the inset of Fig. 7. $P_{\mathrm{C}}$ decreases below and above this optimized bias. The origin of this behavior is still not completely understood at this step and will require further experiments. One possibility to explain the decrease of $P_{\mathrm{C}}$ at low bias would be the complex behavior of the ratio $\tau / \tau_{\mathrm{s}}$ as a function of the applied voltage (linked in particular to an increase of the carrier recombination time $\tau[11,32]$ ). The decrease of $P_{\mathrm{C}}$ at high bias could also be due to the dependence of $\tau / \tau_{\mathrm{s}}$ as a function of $V_{\text {bias }}$, as well as to the DP spin-relaxation mechanism [10] for carriers injected with a large kinetic energy [18]. The best remanent $P_{\mathrm{C}}$ we obtained is already five times higher than the published results using any other PMA injectors [25-29,32]. Although the electrical spin injection efficiency $P_{\mathrm{E}}$ is still lower than the one obtained for the best in-plane injectors [18,20], which could be linked to some particular effect rising from the ultrathin $\mathrm{CoFeB}$ layer during annealing, we believe that detailed interfacial investigation and optimization of the annealing effect could certainly lead to even larger improvement.

\section{CONCLUSIONS}

In conclusion, we have demonstrated a large EL $P_{\mathrm{C}}$ in GaAs-based spin-LED at zero magnetic field with the $\mathrm{CoFeB} / \mathrm{MgO}$ perpendicular spin injector. The value of $P_{\mathrm{C}}$ at remanence is as large as $20 \%$ at $25 \mathrm{~K}$ and still $8 \%$ at $300 \mathrm{~K}$. The electrical spin injection efficiency $P_{\mathrm{E}}$ is found to be almost temperature insensitive at about $16 \pm 4 \%$. In addition, a simple model based on the analysis of the anisotropy of orbital charge occupation and the orbital moment anisotropy is used to explain the origin of PMA at the $\mathrm{MgO} / \mathrm{CoFeB}$ interface and gives a pedagogical and direct image to understand the PMA nature. This demonstration of a robust and efficient remanent electrical spin injection resulting from an ultrathin $\mathrm{CoFeB}$ injector paves the way for future applications based on electrical control of circularly polarized light via SST in III-V spin optronic devices working at RT, as well as for devices where losses due to optical absorption are detrimental, as for example, in spin-injected vertical external cavity surface 
emitting lasers [56], where the magnetic injector would be embedded in the cavity.

\section{ACKNOWLEDGMENTS}

We thank T. Hauet for the help with SQUID measurements and also acknowledge Y. Zheng and D. Demaille for their help with the TEM sample preparation. S.H. Liang acknowledges the joint Ph.D. scholarship from the China Scholarship Council. This work is supported by the joint French National Research Agency (ANR)-National Science Foundation of China (NSFC) SISTER project (Grants No. ANR-11-IS10-0001 and No. NNSFC 61161130527) and French ANR INSPIRE project (Grant No. ANR-10-BLAN-1014).

\section{APPENDIX}

For the TRPL measurements, a mode-locked Ti:sapphire laser (1.5 ps pulse width) was used for the nonresonant circularly polarized excitation at $1.599 \mathrm{eV}$ (i.e., in the GaAs barrier) on a bare $p-i-n$ LED sample without injector. The PL signals were detected by a two-dimensional synchroscan streak camera, which provided an overall temporal resolution less than 8 ps and a spectral resolution of about $8 \mathrm{meV}(5.2 \mathrm{~nm})$. Figure 8(a) shows a typical PL spectra after integration in time domain. Here, we cannot distinguish the multipeak feature as we have observed in EL spectra because the spectral resolution is lower for our PL setup. With a $50 \%$ circularly polarized excitation, we can get about $33 \%$ of $P_{\mathrm{C}}$ for the PL. $P_{\mathrm{C}}$ is almost constant around the maximum of the PL spectra. Then we record the time-resolved information through integrating spectrally around the peak maximum with a window of 4-6 nm, depending on the spectral width. In fact, the dynamic parameters ( $\tau$ and $\tau_{\mathrm{s}}$ ) are not sensitive to the width of this window.

Figure 8(b) (left) shows the PL intensity (color code in arbitrary units) as a function of both time and photon wavelength. The white curve represents the PL intensity $\left(I^{\sigma+}+I^{\sigma-}\right)$ as a function of time when the emission is spectrally integrated. The decay time of this PL intensity
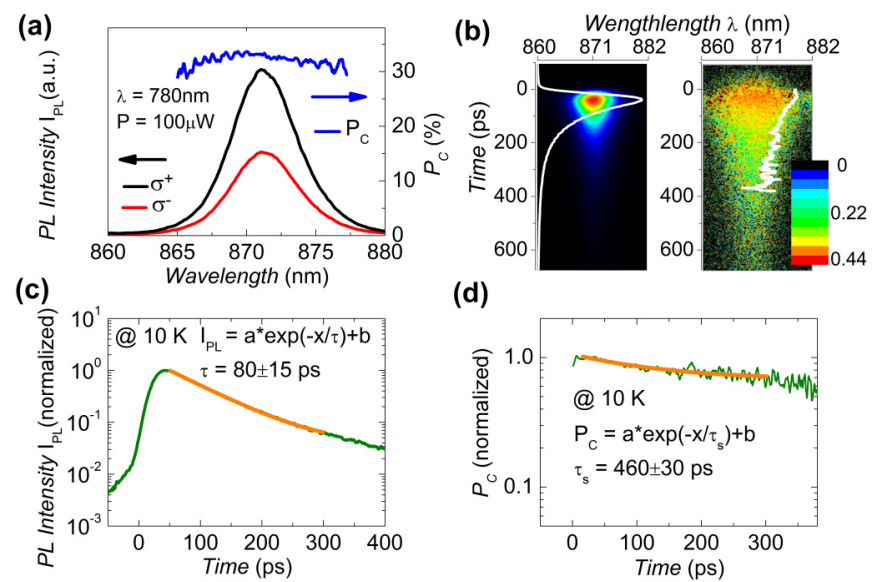

(d)

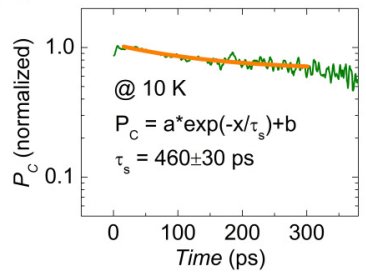

FIG. 8. (Color online) (a) Typical PL spectra after integration in time domain for $\sigma^{+}$and $\sigma^{-}$components. $P_{\mathrm{C}}$ is deduced from $\left(I^{\sigma+}-I^{\sigma-}\right) /\left(I^{\sigma+}+I^{\sigma-}\right)$. (b) Left: PL intensity (color code in arbitrary units) as a function of both time and photon wavelength. The white curve represents the PL intensity as a function of time when the emission is spectrally integrated. Right: PL circular polarization degree $P_{\mathrm{C}}$ (color code from $P_{\mathrm{C}}=0$ to $P_{\mathrm{C}}=0.44$ ) as a function of both time and photon wavelength. The white curve represents $P_{\mathrm{C}}$ as a function of time when the emission is spectrally integrated. (c) Determination of the carrier lifetime $\tau$ from exponential fitting of the decay time of PL intensity $\left(I^{\sigma+}+I^{\sigma-}\right)$ dynamics curve. (d) Determination of the spin lifetime $\tau_{\mathrm{s}}$ from exponential fitting of the decay time of $P_{\mathrm{C}}$ dynamics curve.

corresponds to the carrier lifetime $\tau$. As in the example in Fig. 8(c), we perform the exponential fitting of the PL dynamic curve to obtain $\tau$ to be about $80 \pm 15$ ps. To extract the spin lifetime $\tau_{\mathrm{s}}$, we have studied TRPL circular polarization $P_{\mathrm{C}}$ dynamics. Figure 8 (b) (right) shows $P_{\mathrm{C}}$ (color code from $P_{\mathrm{C}}=0$ to 0.44$)$ as a function of both time and photon wavelength. The white curve represents $P_{\mathrm{C}}$ as a function of time. The decay time of this $P_{\mathrm{C}}$ dynamics that corresponds to the spin relaxation time $\tau_{\mathrm{s}}$ can be extracted by exponential fitting of the curve. As an example shown in Fig. 8(d), the $\tau_{\mathrm{s}}$ can be determined to be about $460 \pm 30$ ps.
[1] R. Fiederling, M. Keim, G. Reuscher, W. Ossau, G. Schmidt, A. Waag, and L. W. Molenkamp, Nature (London) 402, 787 (1999).

[2] Y. Ohno, D. K. Young, B. Beschoten, F. Matsukura, H. Ohno, and D. D. Awschalom, Nature (London) 402, 790 (1999).

[3] T. D. Nguyen, E. Ehrenfreund, and Z. V. Vardeny, Science 337, 204 (2012).

[4] R. Farshchi, M. Ramsteiner, J. Herfort, A. Tahraoui, and H. T. Grahn, Appl. Phys. Lett. 98, 162508 (2011).

[5] M. Holub and P. Bhattacharya, J. Phys. D: Appl. Phys. 40, R179 (2007).

[6] P. Asshoff, A. Merz, H. Kalt, and M. Hetterich, Appl. Phys. Lett. 98, 112106 (2011).
[7] J. Xu, A. Lakhtakia, J. Liou, A. Chen, and I. J. Hodgkinson, Opt. Commun. 264, 235 (2006).

[8] D. Y. Kim, J. Korean Phys. Soc. 49, S505 (2006).

[9] F. Meier and B. P. Zakharchenya, Optical Orientation (NorthHolland, Amsterdam, The Netherlands, 1984.)

[10] M. I. Dyakonov, Spin Physics in Semiconductors, (Springer, Berlin, Germany, 2008)

[11] C. Adelmann, X. Lou, J. Strand, C. J. Palmstrøm, and P. A. Crowell, Phys. Rev. B 71, 121301(R) (2005).

[12] X. Y. Dong, C. Adelmann, J. Q. Xie, C. J. Palmstrøm, X. Lou, J. Strand, P. A. Crowell, J.-P. Barnes, and A. K. Petford-Long, Appl. Phys. Lett. 86, 102107 (2005).

[13] M.-S. Choi, F. Plastina, and R. Fazio, Phys. Rev. Lett. 87, 116601 (2001). 
[14] A. T. Hanbicki, B. T. Jonker, G. Itskos, G. Kioseoglou, and A. Petrou, Appl. Phys. Lett. 80, 1240 (2002).

[15] A. T. Hanbicki, O. M. J. van't Erve, R. Magno, G. Kioseoglou, C. H. Li, B. T. Jonker, G. Itskos, R. Mallory, M. Yasar, and A. Petrou, Appl. Phys. Lett. 82, 4092 (2003).

[16] O. M. J. van't Erve, G. Kioseoglou, A. T. Hanbicki, C. H. Li, B. T. Jonker, R. Mallory, M. Yasar, and A. Petrou, Appl. Phys. Lett. 84, 4334 (2004).

[17] B. T. Jonker, G. Kioseoglou, A. T. Hanbicki, C. H. Li, and P. E. Thompson, Nat. Phys. 3, 542 (2007).

[18] X. Jiang, R. Wang, R. M. Shelby, R. M. Macfarlane, S. R. Bank, J. S. Harris, and S. S. P. Parkin, Phys. Rev. Lett. 94, 056601 (2005).

[19] L. Lombez, P. Renucci, P. F. Braun, H. Carrère, X. Marie, T. Amand, B. Urbaszek, J. L. Gauffier, P. Gallo, T. Camps, A. Arnoult, C. Fontaine, C. Deranlot, R. Mattana, H. Jaffrès, J.-M. George, and P. H. Binh, Appl. Phys. Lett. 90, 081111 (2007).

[20] Y. Lu, V. G. Truong, P. Renucci, M. Tran, H. Jaffrès, C. Deranlot, J.-M. George, A. Lemaître, Y. Zheng, D. Demaille, P.-H. Binh, T. Amand, and X. Marie, Appl. Phys. Lett. 93, 152102 (2008).

[21] V. G. Truong, P. H. Binh, P. Renucci, M. Tran, H. Jaffrès, Y. Lu, C. Deranlot, J.-M. George, A. Lemaître, T. Amand, and X. Marie, Appl. Phys. Lett. 94, 141109 (2009).

[22] E. D. Fraser, S. Hegde, L. Schweidenback, A. H. Russ, A. Petrou, H. Luo, and G. Kioseoglou, Appl. Phys. Lett. 97, 041103 (2010).

[23] N. Nishizawa and H. Munekata, J. Appl. Phys. 114, 033507 (2013).

[24] O. M. J. van’t Erve, G. Kioseoglou, A. T. Hanbicki, C. H. Li, and B. T. Jonker, Appl. Phys. Lett. 89, 072505 (2006).

[25] N. C. Gerhardt, S. Hövel, C. Brenner, M. R. Hofmann, F.-Y. Lo, D. Reuter, A. D. Wieck, E. Schuster, W. Keune, and K. Westerholt, Appl. Phys. Lett. 87, 032502 (2005).

[26] S. Hövel, N. C. Gerhardt, M. R. Hofmann, F.-Y. Lo, A. Ludwig, D. Reuter, A. D. Wieck, E. Schuster, H. Wende, W. Keune, O. Petracic, and K. Westerholt, Appl. Phys. Lett. 93, 021117 (2008).

[27] A. Sinsarp, T. Manago, F. Takano, and H. Akinaga, J. Supercond. Nov. Magn. 20, 405 (2007).

[28] L. Grenet, M. Jamet, P. Noé, V. Calvo, J.-M. Hartmann, L. E. Nistor, B. Rodmacq, S. Auffret, P. Warin, and Y. Samson, Appl. Phys. Lett. 94, 032502 (2009).

[29] J. Zarpellon, H. Jaffrès, J. Frougier, C. Deranlot, J. M. George, D. H. Mosca, A. Lemaître, F. Freimuth, Q. H. Duong, P. Renucci, and X. Marie, Phys. Rev. B 86, 205314 (2012).

[30] S. Girod, M. Gottwald, S. Andrieu, S. Mangin, J. McCord, Eric E. Fullerton, J.-M. L. Beaujour, B. J. Krishnatreya, and A. D. Kent, Appl. Phys. Lett. 94, 262504 (2009).

[31] A. Fert and H. Jaffrès, Phys. Rev. B 64, 184420 (2001).

[32] C. Adelmann, J. L. Hilton, B. D. Schultz, S. McKernan, C. J. Palmstrøm, X. Lou, H.-S. Chiang, and P. A. Crowell, Appl. Phys. Lett. 89, 112511 (2006).

[33] H. X. Yang, M. Chshiev, B. Dieny, J. H. Lee, A. Manchon, and K. H. Shin, Phys. Rev. B 84, 054401 (2011).
[34] K. H. Khoo, G. Wu, M. H. Jhon, M. Tran, F. Ernult, K. Eason, H. J. Choi, and C. K. Gan, Phys. Rev. B 87, 174403 (2013).

[35] S. Ikeda, K. Miura, H. Yamamoto, K. Mizunuma, H. D. Gan, M. Endo, S. Kanai, J. Hayakawa, F. Matsukura, and H. Ohno, Nat. Mater. 9, 721 (2010).

[36] D. C. Worledge, G. Hu, D. W. Abraham, J. Z. Sun, P. L. Trouilloud, J. Nowak, S. Brown, M. C. Gaidis, E. J. O’Sullivan, and R. P. Robertazzi, Appl. Phys. Lett. 98, 022501 (2011).

[37] W. G. Wang, S. Hageman, M. Li, S. X. Huang, X. M. Kou, X. Fan, John Q. Xiao, and C. L. Chien, Appl. Phys. Lett. 99, $102502(2011)$.

[38] W. X. Wang, Y. Yang, H. Naganuma, Y. Ando, R. C. Yu, and X. F. Han, Appl. Phys. Lett. 99, 012502 (2011).

[39] T. Zhu, Y. Yang, R. C. Yu, H. Ambaye, V. Lauter, and J. Q. Xiao, Appl. Phys. Lett. 100, 202406 (2012).

[40] Y. Jang, C. Nam, K.-S. Lee, B. K. Cho, Y. J. Cho, K.-S. Kim, and K. W. Kim, Appl. Phys. Lett. 91, 102104 (2007).

[41] H. Meng, W. H. Lum, R. Sbiaa, S. Y. H. Lua, and H. K. Tan, J. Appl. Phys. 110, 033904 (2011).

[42] K. Nakamura, T. Akiyama, T. Ito, M. Weinert, and A. J. Freeman, Phys. Rev. B 81, 220409(R) (2010).

[43] G. Kresse and J. Hafner, Phys. Rev. B 47, 558 (1993).

[44] G. Kresse and J. Furthmüller, Phys. Rev. B 54, 11169 (1996).

[45] G. Kresse and J. Furthmüller, Comput. Mater. Sci. 6, 15 (1996).

[46] Y. Wang and J. P. Perdew, Phys. Rev. B 44, 13298 (1991).

[47] P. E. Blöchl, Phys. Rev. B 50, 17953 (1994).

[48] G. Kresse and D. Joubert, Phys. Rev. B 59, 1758 (1999).

[49] P. Bruno, Phys. Rev. B 39, 865 (1989).

[50] One cannot totally exclude the contribution of a bound exciton at $25 \mathrm{~K}$ (it can be ruled out at higher temperature). However, this contribution would only result in an artificial diminution of the EL circular polarization compared to the one of the free exciton line Refs. [51,52].

[51] B. T. Jonker, A. T. Hanbicki, Y. D. Park, G. Itskos, M. Furis, G. Kioseoglou, A. Petrou, and X. Wei, Appl. Phys. Lett. 79, 3098 (2001).

[52] R. Mallory, M. Yasar, G. Itskos, A. Petrou, G. Kioseoglou, A. T. Hanbicki, C. H. Li, O. M. J. van't Erve, B. T. Jonker, M. Shen, and S. Saikin, Phys. Rev. B 73, 115308 (2006).

[53] L. Lombez, P.-F. Braun, H. Carrère, B. Urbaszek, P. Renucci, T. Amand, X. Marie, J. C. Harmand, and V. K. Kalevich, Appl. Phys. Lett. 87, 252115 (2005).

[54] G. Salis, R. Wang, X. Jiang, R. M. Shelby, S. S. Parkin, S. R. Bank, and J. S. Harris, Appl. Phys. Lett. 87, 262503 (2005).

[55] H. Soldat, M. Li, N. C. Gerhardt, M. R. Hofmann, A. Ludwig, A. Ebbing, D. Reuter, A. D. Wieck, F. Stromberg, W. Keune, and H. Wende, Appl. Phys. Lett. 99, 051102 (2011).

[56] J. Frougier, G. Baili, M. Alouini, I. Sagnes, H. Jaffrès, A. Garnache, C. Deranlot, D. Dolfi, and J. M. George, Appl. Phys. Lett. 103, 252402 (2013). 